Research Article

\title{
Boundedness of Vector-Valued Intrinsic Square Functions in Morrey Type Spaces
}

\author{
Hua Wang \\ College of Mathematics and Econometrics, Hunan University, Changsha 410082, China \\ Correspondence should be addressed to Hua Wang; wanghua@pku.edu.cn \\ Received 4 December 2013; Accepted 11 April 2014; Published 30 April 2014 \\ Academic Editor: Lars E. Persson
}

Copyright (c) 2014 Hua Wang. This is an open access article distributed under the Creative Commons Attribution License, which permits unrestricted use, distribution, and reproduction in any medium, provided the original work is properly cited.

We will obtain the strong type and weak type estimates for vector-valued analogues of intrinsic square functions in the weighted Morrey spaces $L^{p, \kappa}(w)$ when $1 \leq p<\infty, 0<\kappa<1$, and in the generalized Morrey spaces $L^{p, \Phi}$ for $1 \leq p<\infty$, where $\Phi$ is a growth function on $(0, \infty)$ satisfying the doubling condition.

\section{Introduction and Main Results}

The intrinsic square functions were first introduced by Wilson in [1,2]; they are defined as follows. For $0<\alpha \leq 1$, let $\mathscr{C}_{\alpha}$ be the family of functions $\varphi$ defined on $\mathbb{R}^{n}$ such that $\varphi$ has support containing in $\left\{x \in \mathbb{R}^{n}:|x| \leq 1\right\}, \int_{\mathbb{R}^{n}} \varphi(x) d x=0$, and, for all $x, x^{\prime} \in \mathbb{R}^{n}$,

$$
\left|\varphi(x)-\varphi\left(x^{\prime}\right)\right| \leq\left|x-x^{\prime}\right|^{\alpha} .
$$

For $(y, t) \in \mathbb{R}_{+}^{n+1}=\mathbb{R}^{n} \times(0, \infty)$ and $f \in L_{\text {loc }}^{1}\left(\mathbb{R}^{n}\right)$, we set

$$
\begin{aligned}
A_{\alpha}(f)(y, t) & =\sup _{\varphi \in \mathscr{C}_{\alpha}}\left|f * \varphi_{t}(y)\right| \\
& =\sup _{\varphi \in \mathscr{C}_{\alpha}}\left|\int_{\mathbb{R}^{n}} \varphi_{t}(y-z) f(z) d z\right| .
\end{aligned}
$$

Then we define the intrinsic square function of $f$ (of order $\alpha$ ) by

$$
\mathcal{S}_{\alpha}(f)(x)=\left(\iint_{\Gamma(x)}\left(A_{\alpha}(f)(y, t)\right)^{2} \frac{d y d t}{t^{n+1}}\right)^{1 / 2}
$$

where $\Gamma(x)$ denotes the usual cone of aperture one:

$$
\Gamma(x)=\left\{(y, t) \in \mathbb{R}_{+}^{n+1}:|x-y|<t\right\} .
$$

Let $\vec{f}=\left(f_{1}, f_{2}, \ldots\right)$ be a sequence of locally integrable functions on $\mathbb{R}^{n}$. For any $x \in \mathbb{R}^{n}$, Wilson [2] also defined the vector-valued intrinsic square functions of $\vec{f}$ by

$$
\mathcal{S}_{\alpha}(\vec{f})(x)=\left(\sum_{j=1}^{\infty}\left|\mathcal{S}_{\alpha}\left(f_{j}\right)(x)\right|^{2}\right)^{1 / 2} .
$$

In [2], Wilson has established the following two theorems.

Theorem A (see [2]). Let $0<\alpha \leq 1,1<p<\infty$, and $w \in A_{p}$ (Muckenhoupt weight class). Then there exists a constant $C>0$ independent of $\vec{f}=\left(f_{1}, f_{2}, \ldots\right)$ such that

$$
\left\|\left(\sum_{j}\left|\delta_{\alpha}\left(f_{j}\right)\right|^{2}\right)^{1 / 2}\right\|_{L_{w}^{p}} \leq C\left\|\left(\sum_{j}\left|f_{j}\right|^{2}\right)^{1 / 2}\right\|_{L_{w}^{p}} .
$$

Theorem $\overline{\mathrm{B}}$ (see [2]). Let $0<\alpha \leq 1$ and $p=1$. Then, for any given weight function $w$ and $\lambda>0$, there exists a constant $C>0$ independent of $\vec{f}=\left(f_{1}, f_{2}, \ldots\right)$ and $\lambda$ such that

$$
w\left(\left\{x \in \mathbb{R}^{n}:\left(\sum_{j}\left|\mathcal{\delta}_{\alpha}\left(f_{j}\right)(x)\right|^{2}\right)^{1 / 2}>\lambda\right\}\right)
$$

$$
\leq \frac{C}{\lambda} \int_{\mathbb{R}^{n}}\left(\sum_{j}\left|f_{j}(x)\right|^{2}\right)^{1 / 2} M w(x) d x,
$$


where $M$ denotes the standard Hardy-Littlewood maximal operator.

If we take $w \in A_{1}$, then $M(w)(x) \leq C \cdot w(x)$ for a.e. $x \in \mathbb{R}^{n}$ by the definition of $A_{1}$ weights (see Section 2 ). Hence, as a straightforward consequence of Theorem $\bar{B}$, we obtain the following.

Theorem B. Let $0<\alpha \leq 1, p=1$, and $w \in A_{1}$. Then there exists a constant $C>0$ independent of $\vec{f}=\left(f_{1}, f_{2}, \ldots\right)$ such that

$$
\left\|\left(\sum_{j}\left|\mathcal{S}_{\alpha}\left(f_{j}\right)\right|^{2}\right)^{1 / 2}\right\|_{W L_{w}^{1}} \leq C\left\|\left(\sum_{j}\left|f_{j}\right|^{2}\right)^{1 / 2}\right\|_{L_{w}^{1}} .
$$

In particular, if we take $w$ to be a constant function, then we immediately get the following.

Theorem C. Let $0<\alpha \leq 1$ and $1<p<\infty$. Then there exists a constant $C>0$ independent of $\vec{f}=\left(f_{1}, f_{2}, \ldots\right)$ such that

$$
\left\|\left(\sum_{j}\left|\mathcal{S}_{\alpha}\left(f_{j}\right)\right|^{2}\right)^{1 / 2}\right\|_{L^{p}} \leq C\left\|\left(\sum_{j}\left|f_{j}\right|^{2}\right)^{1 / 2}\right\|_{L^{p}} .
$$

Theorem D. Let $0<\alpha \leq 1$ and $p=1$. Then there exists $a$ constant $C>0$ independent of $\vec{f}=\left(f_{1}, f_{2}, \ldots\right)$ such that

$$
\left\|\left(\sum_{j}\left|\mathcal{S}_{\alpha}\left(f_{j}\right)\right|^{2}\right)^{1 / 2}\right\|_{W L^{1}} \leq C\left\|\left(\sum_{j}\left|f_{j}\right|^{2}\right)^{1 / 2}\right\|_{L^{1}} .
$$

On the other hand, the classical Morrey spaces $\mathscr{L}^{p, \lambda}$ were originally introduced by Morrey in [3] to study the local behavior of solutions to second order elliptic partial differential equations. Since then, these spaces play an important role in studying the regularity of solutions to partial differential equations. For the boundedness of the HardyLittlewood maximal operator, the fractional integral operator, and the Calderón-Zygmund singular integral operator on these spaces, we refer the reader to [4-6]. In [7], Mizuhara introduced the generalized Morrey spaces $L^{p, \Phi}$ which was later extended and studied by many authors (see [8-12]). In [13], Komori and Shirai defined the weighted Morrey spaces $L^{p, \kappa}(w)$ which could be viewed as an extension of weighted Lebesgue spaces and then discussed the boundedness of the above classical operators in harmonic analysis on these weighted spaces. Recently, in [14-16], we have established the strong type and weak type estimates for intrinsic square functions on $L^{p, \Phi}$ and $L^{p, \kappa}(w)$.

For the boundedness of vector-valued intrinsic square functions in the weighted Morrey spaces $L^{p, \kappa}(w)$ for all $1 \leq$ $p<\infty$ and $0<\kappa<1$, we will prove the following.
Theorem 1. Let $0<\alpha \leq 1,1<p<\infty, 0<\kappa<1$, and $w \in A_{p}$. Then there is a constant $C>0$ independent of $\vec{f}=$ $\left(f_{1}, f_{2}, \ldots\right)$ such that

$$
\left\|\left(\sum_{j}\left|\mathcal{S}_{\alpha}\left(f_{j}\right)\right|^{2}\right)^{1 / 2}\right\|_{L^{p, \kappa}(w)} \leq C\left\|\left(\sum_{j}\left|f_{j}\right|^{2}\right)^{1 / 2}\right\|_{L^{p, \kappa}(w)} .
$$

Theorem 2. Let $0<\alpha \leq 1, p=1,0<\kappa<1$, and $w \in A_{1}$. Then there is a constant $C>0$ independent of $\vec{f}=\left(f_{1}, f_{2}, \ldots\right)$ such that

$$
\left\|\left(\sum_{j}\left|\mathcal{S}_{\alpha}\left(f_{j}\right)\right|^{2}\right)^{1 / 2}\right\|_{W L^{1, \kappa}(w)} \leq C\left\|\left(\sum_{j}\left|f_{j}\right|^{2}\right)^{1 / 2}\right\|_{L^{1, \kappa}(w)} .
$$

For the continuity properties of $\mathcal{S}_{\alpha}(\vec{f})$ in $L^{p, \Phi}$ for all $1 \leq$ $p<\infty$, we will show the following.

Theorem 3. Let $0<\alpha \leq 1$ and $1<p<\infty$. Assume that $\Phi$ satisfies (15) and $1 \leq D(\Phi)<2^{n}$; then there is a constant $C>0$ independent of $\vec{f}=\left(f_{1}, f_{2}, \ldots\right)$ such that

$$
\left\|\left(\sum_{j}\left|\delta_{\alpha}\left(f_{j}\right)\right|^{2}\right)^{1 / 2}\right\|_{L^{p, \Phi}} \leq C\left\|\left(\sum_{j}\left|f_{j}\right|^{2}\right)^{1 / 2}\right\|_{L^{p, \Phi}} .
$$

Theorem 4. Let $0<\alpha \leq 1$ and $p=1$. Assume that $\Phi$ satisfies (15) and $1 \leq D(\Phi)<2^{n}$; then there is a constant $C>0$ independent of $\vec{f}=\left(f_{1}, f_{2}, \ldots\right)$ such that

$$
\left\|\left(\sum_{j}\left|\delta_{\alpha}\left(f_{j}\right)\right|^{2}\right)^{1 / 2}\right\|_{W L^{1, \Phi}} \leq C\left\|\left(\sum_{j}\left|f_{j}\right|^{2}\right)^{1 / 2}\right\|_{L^{1, \Phi}} .
$$

\section{Notations and Definitions}

2.1. Generalized Morrey Spaces. Let $\Phi=\Phi(r), r>0$, be a growth function, that is, a positive increasing function in $(0, \infty)$, and satisfy the following doubling condition:

$$
\Phi(2 r) \leq D \cdot \Phi(r), \quad \forall r>0,
$$

where $D=D(\Phi) \geq 1$ is a doubling constant independent of $r$.

Definition 5 (see [7]). Let $1 \leq p<\infty$. We denote by $L^{p, \Phi}=L^{p, \Phi}\left(\mathbb{R}^{n}\right)$ the space of all locally integrable functions $f$ defined on $\mathbb{R}^{n}$, such that for every $x_{0} \in \mathbb{R}^{n}$ and all $r>0$

$$
\int_{B\left(x_{0}, r\right)}|f(x)|^{p} d x \leq C^{p} \Phi(r)
$$

where $B\left(x_{0}, r\right)=\left\{x \in \mathbb{R}^{n}:\left|x-x_{0}\right|<r\right\}$ is the ball centered at $x_{0}$ and with radius $r>0$. Then we let $\|f\|_{L^{p, \Phi}}$ be the smallest constant $C>0$ satisfying (16) and $L^{p, \Phi}\left(\mathbb{R}^{n}\right)$ becomes a Banach space with norm $\|\cdot\|_{L^{p, \Phi}}$. 
Obviously, when $\Phi(r)=r^{\lambda}$ with $0<\lambda<n, L^{p, \Phi}$ is just the classical Morrey spaces introduced in [3]. We also denote by $W L^{1, \Phi}=W L^{1, \Phi}\left(\mathbb{R}^{n}\right)$ the generalized weak Morrey spaces of all measurable functions $f$ for which

$$
\sup _{\lambda>0} \lambda \cdot\left|\left\{x \in B\left(x_{0}, r\right):|f(x)|>\lambda\right\}\right| \leq C \Phi(r),
$$

for every $x_{0} \in \mathbb{R}^{n}$ and all $r>0$. The smallest constant $C>0$ satisfying (17) is also denoted by $\|f\|_{W L^{1, \Phi}}$.

2.2. Weighted Morrey Spaces. A weight $w$ is a positive, locally integrable function on $\mathbb{R}^{n} ; B=B\left(x_{0}, r_{B}\right)$ denotes the ball with the center $x_{0}$ and radius $r_{B}$. Given a ball $B$ and $\lambda>0$, $\lambda B$ denotes the ball with the same center as $B$ whose radius is $\lambda$ times that of $B$. For a given weight function $w$ and a measurable set $E$, we also denote the Lebesgue measure of $E$ by $|E|$ and the weighted measure of $E$ by $w(E)$, where $w(E)=\int_{E} w(x) d x$. For $1<p<\infty$, a weight function $w$ is said to belong to $A_{p}$, if there is a constant $C>0$ such that, for every ball $B \subseteq \mathbb{R}^{n}$,

$$
\left(\frac{1}{|B|} \int_{B} w(x) d x\right)\left(\frac{1}{|B|} \int_{B} w(x)^{-1 /(p-1)} d x\right)^{p-1} \leq C .
$$

For the case $p=1, w \in A_{1}$, if there is a constant $C>0$ such that, for every ball $B \subseteq \mathbb{R}^{n}$,

$$
\frac{1}{|B|} \int_{B} w(x) d x \leq C \cdot \operatorname{ess} \inf _{x \in B} w(x) .
$$

A weight function $w \in A_{\infty}$ if it satisfies the $A_{p}$ condition for some $1 \leq p<\infty$. It is well known that if $w \in A_{p}$ with $1 \leq p<\infty$, then, for any ball $B$, there exists an absolute constant $C>0$ such that

$$
w(2 B) \leq C w(B) .
$$

Moreover, if $w \in A_{\infty}$, then, for all balls $B$ and all measurable subsets $E$ of $B$, there exists a number $\delta>0$ independent of $E$ and $B$ such that

$$
\frac{w(E)}{w(B)} \leq C\left(\frac{|E|}{|B|}\right)^{\delta} .
$$

Given a weight function $w$ on $\mathbb{R}^{n}$, for $1 \leq p<\infty$, the weighted Lebesgue space $L_{w}^{p}\left(\mathbb{R}^{n}\right)$ is defined as the set of all functions $f$ such that

$$
\|f\|_{L_{w}^{p}}=\left(\int_{\mathbb{R}^{n}}|f(x)|^{p} w(x) d x\right)^{1 / p}<\infty .
$$

We also denote by $W L_{w}^{1}\left(\mathbb{R}^{n}\right)$ the weighted weak space consisting of all measurable functions $f$ such that

$$
\|f\|_{W L_{w}^{1}}=\sup _{\lambda>0} \lambda \cdot w\left(\left\{x \in \mathbb{R}^{n}:|f(x)|>\lambda\right\}\right)<\infty .
$$

In particular, when $w$ equals to a constant function, we will denote $L_{w}^{p}\left(\mathbb{R}^{n}\right)$ and $W L_{w}^{1}\left(\mathbb{R}^{n}\right)$ simply by $L^{p}\left(\mathbb{R}^{n}\right)$ and $W L^{1}\left(\mathbb{R}^{n}\right)$.
Definition 6 (see [13]). Let $1 \leq p<\infty, 0<\kappa<1$, and $w$ be a weight function on $\mathbb{R}^{n}$. Then the weighted Morrey space is defined by

$$
L^{p, \kappa}(w)=\left\{f \in L_{\mathrm{loc}}^{p}(w):\|f\|_{L^{p, \kappa}(w)}<\infty\right\},
$$

where

$$
\|f\|_{L^{p, \kappa}(w)}=\sup _{B}\left(\frac{1}{w(B)^{\kappa}} \int_{B}|f(x)|^{p} w(x) d x\right)^{1 / p}
$$

and the supremum is taken over all balls $B$ in $\mathbb{R}^{n}$.

For $p=1$ and $0<\kappa<1$, we also denote by $W L^{1, \kappa}(w)$ the weighted weak Morrey spaces of all measurable functions $f$ satisfying

$$
\begin{aligned}
\|f\|_{W L^{1, \kappa}(w)}= & \sup _{B} \sup _{\lambda>0} \frac{1}{w(B)^{\kappa}} \lambda \\
& \cdot w(\{x \in B:|f(x)|>\lambda\})<\infty .
\end{aligned}
$$

Throughout this paper, the letter $C$ always denotes a positive constant independent of the main parameters involved, but it may be different from line to line.

\section{Proofs of Theorems 1 and 2}

Proof of Theorem 1. Let $\left(\sum_{j}\left|f_{j}\right|^{2}\right)^{1 / 2} \in L^{p, \kappa}(w)$ with $1<p<$ $\infty$ and $0<\kappa<1$. Fix a ball $B=B\left(x_{0}, r_{B}\right) \subseteq \mathbb{R}^{n}$ and decompose $f_{j}=f_{j}^{0}+f_{j}^{\infty}$, where $f_{j}^{0}=f_{j} \cdot \chi_{2 B}$ and $\chi_{2 B}$ denotes the characteristic function of $2 B=B\left(x_{0}, 2 r_{B}\right), j=1,2, \ldots$. Then we write

$$
\begin{aligned}
& \frac{1}{w(B)^{\kappa / p}}\left(\int_{B}\left(\sum_{j}\left|\mathcal{S}_{\alpha}\left(f_{j}\right)(x)\right|^{2}\right)^{p / 2} w(x) d x\right)^{1 / p} \\
& \leq \frac{1}{w(B)^{\kappa / p}}\left(\int_{B}\left(\sum_{j}\left|\mathcal{S}_{\alpha}\left(f_{j}^{0}\right)(x)\right|^{2}\right)^{p / 2} w(x) d x\right)^{1 / p} \\
& \quad+\frac{1}{w(B)^{\kappa / p}}\left(\int_{B}\left(\sum_{j}\left|\mathcal{S}_{\alpha}\left(f_{j}^{\infty}\right)(x)\right|^{2}\right)^{p / 2} w(x) d x\right)^{1 / p} \\
& =I_{1}+I_{2} .
\end{aligned}
$$


Using Theorem A and inequality (20), we have

$$
\begin{aligned}
I_{1} & \leq \frac{1}{w(B)^{\kappa / p}}\left\|\left(\sum_{j}\left|\mathcal{S}_{\alpha}\left(f_{j}^{0}\right)\right|^{2}\right)^{1 / 2}\right\|_{L_{w}^{p}} \\
& \leq C \cdot \frac{1}{w(B)^{\kappa / p}}\left(\int_{2 B}\left(\sum_{j}\left|f_{j}(x)\right|^{2}\right)^{p / 2} w(x) d x\right)^{1 / p} \\
& \leq C\left\|\left(\sum_{j}\left|f_{j}\right|^{2}\right)^{1 / 2}\right\|_{L^{p, \kappa}(w)} \cdot \frac{w(2 B)^{\kappa / p}}{w(B)^{\kappa / p}} \\
& \leq C\left\|\left(\sum_{j}\left|f_{j}\right|^{2}\right)^{1 / 2}\right\|_{L^{p, \kappa}(w)} \cdot
\end{aligned}
$$

Let us now turn to estimate the other term $I_{2}$. For any $\varphi \in \mathscr{C}_{\alpha}$, $0<\alpha \leq 1, j=1,2, \ldots$, and $(y, t) \in \Gamma(x)$, we have

$$
\begin{aligned}
\left|f_{j}^{\infty} * \varphi_{t}(y)\right| & =\left|\int_{(2 B)^{c}} \varphi_{t}(y-z) f_{j}(z) d z\right| \\
& \leq C \cdot t^{-n} \int_{(2 B)^{c} \cap\{z:|y-z| \leq t\}}\left|f_{j}(z)\right| d z \\
& \leq C \cdot t^{-n} \sum_{\ell=1}^{\infty} \int_{\left(2^{\ell+1} B \backslash 2^{\ell} B\right) \cap\{z:|y-z| \leq t\}}\left|f_{j}(z)\right| d z .
\end{aligned}
$$

For any $x \in B,(y, t) \in \Gamma(x)$, and $z \in\left(2^{\ell+1} B \backslash 2^{\ell} B\right) \cap B(y, t)$, then, by a direct computation, we can easily see that

$$
\begin{aligned}
2 t & \geq|x-y|+|y-z| \geq|x-z| \\
& \geq\left|z-x_{0}\right|-\left|x-x_{0}\right| \geq 2^{\ell-1} r_{B}
\end{aligned}
$$

Thus, by using the above inequalities (29) and (30), together with Minkowski's inequality for integrals, we deduce

$$
\begin{aligned}
& \left|\mathcal{S}_{\alpha}\left(f_{j}^{\infty}\right)(x)\right| \\
& \quad=\left(\iint_{\Gamma(x)} \sup _{\varphi \in \mathscr{C}_{\alpha}}\left|f_{j}^{\infty} * \varphi_{t}(y)\right|^{2} \frac{d y d t}{t^{n+1}}\right)^{1 / 2} \\
& \leq C\left(\int_{2^{\ell-2} r_{B}}^{\infty} \int_{|x-y|<t}\left|t^{-n} \sum_{\ell=1}^{\infty} \int_{2^{\ell+1} B \backslash 2^{\ell} B}\right| f_{j}(z)|d z|^{2} \frac{d y d t}{t^{n+1}}\right)^{1 / 2} \\
& \leq C\left(\sum_{\ell=1}^{\infty} \int_{2^{\ell+1} B \backslash 2^{\ell} B}\left|f_{j}(z)\right| d z\right)\left(\int_{2^{\ell-2} r_{B}}^{\infty} \frac{d t}{t^{2 n+1}}\right)^{1 / 2} \\
& \leq C \sum_{\ell=1}^{\infty} \frac{1}{\left|2^{\ell+1} B\right|} \int_{2^{\ell+1} B \backslash 2^{\ell} B}\left|f_{j}(z)\right| d z .
\end{aligned}
$$

Then, by duality and Cauchy-Schwarz inequality, we get

$$
\begin{aligned}
& \left(\sum_{j}\left|\mathcal{S}_{\alpha}\left(f_{j}^{\infty}\right)(x)\right|^{2}\right)^{1 / 2} \\
& \leq C\left(\sum_{j}\left|\sum_{\ell=1}^{\infty} \frac{1}{\left|2^{\ell+1} B\right|} \int_{2^{\ell+1} B \mid 2^{\ell} B}\right| f_{j}(z)|d z|^{2}\right)^{1 / 2} \\
& \leq C \sup _{\left(\sum_{j}\left|\zeta_{j}\right|^{2}\right)^{1 / 2} \leq 1} \sum_{j}\left(\sum_{\ell=1}^{\infty} \frac{1}{\left|2^{\ell+1} B\right|} \int_{2^{\ell+1} B}\left|f_{j}(z)\right| d z \cdot \zeta_{j}\right) \\
& \leq C \sum_{\ell=1}^{\infty} \frac{1}{\left|2^{\ell+1} B\right|} \int_{2^{\ell+1} B}\left(\sum_{j}\left|\zeta_{j}\right|^{2}\right)^{1 / 2} \leq 1 \\
& \leq C \sum_{\ell=1}^{\infty} \frac{1}{\left|2^{\ell+1} B\right|} \int_{2^{\ell+1} B}\left(\sum_{j}\left|f_{j}(z)\right|^{2}\right)^{1 / 2} d z .
\end{aligned}
$$

Furthermore, it follows from Hölder's inequality, (32), and the $A_{p}$ condition that

$$
\begin{aligned}
& \left(\sum_{j}\left|\mathcal{S}_{\alpha}\left(f_{j}^{\infty}\right)(x)\right|^{2}\right)^{1 / 2} \\
& \leq C \sum_{\ell=1}^{\infty} \frac{1}{\left|2^{\ell+1} B\right|}\left(\int_{2^{\ell+1} B}\left(\sum_{j}\left|f_{j}(z)\right|^{2}\right)^{p / 2} w(z) d z\right)^{1 / p} \\
& \quad \times\left(\int_{2^{\ell+1} B} w(z)^{-p^{\prime} / p} d z\right)^{1 / p^{\prime}} \\
& \leq C\left\|\left(\sum_{j}\left|f_{j}\right|^{2}\right)^{1 / 2}\right\|_{L^{p, \kappa}(w)} \cdot \sum_{\ell=1}^{\infty} w\left(2^{\ell+1} B\right)^{(\kappa-1) / p}
\end{aligned}
$$

where we denote the conjugate exponent of $p>1$ by $p^{\prime}=$ $p /(p-1)$. Note that $w \in A_{p} \subset A_{\infty}$ for all $1<p<\infty$. Hence, we apply inequality (21) to obtain

$$
\begin{aligned}
I_{2} & \leq C\left\|\left(\sum_{j}\left|f_{j}\right|^{2}\right)^{1 / 2}\right\|_{L^{p, \kappa}(w)} \cdot \sum_{\ell=1}^{\infty} \frac{w(B)^{(1-\kappa) / p}}{w\left(2^{\ell+1} B\right)^{(1-\kappa) / p}} \\
& \leq C\left\|\left(\sum_{j}\left|f_{j}\right|^{2}\right)^{1 / 2}\right\|_{L^{p, \kappa}(w)} \cdot \sum_{\ell=1}^{\infty}\left(\frac{|B|}{\left|2^{\ell+1} B\right|}\right)^{\delta \cdot(1-\kappa) / p} \\
& \leq C\left\|\left(\sum_{j}\left|f_{j}\right|^{2}\right)^{1 / 2}\right\|_{L^{p, \kappa}(w)},
\end{aligned}
$$

where the last series is convergent since $0<\kappa<1$ and $\delta>0$. Summarizing the above two estimates for $I_{1}$ and $I_{2}$ and then taking the supremum over all balls $B \subseteq \mathbb{R}^{n}$, we complete the proof of Theorem 1 . 
Proof of Theorem 2. Let $\left(\sum_{j}\left|f_{j}\right|^{2}\right)^{1 / 2} \in L^{1, \kappa}(w)$ with $0<\kappa<$ 1 . Fix a ball $B=B\left(x_{0}, r_{B}\right) \subseteq \mathbb{R}^{n}$; we set $f_{j}=f_{j}^{0}+f_{j}^{\infty}$, where $f_{j}^{0}=f_{j} \cdot \chi_{2 B}, j=1,2, \ldots$. Then, for any given $\lambda>0$, one writes

$$
\begin{aligned}
& w\left(\left\{x \in B:\left(\sum_{j}\left|\mathcal{S}_{\alpha}\left(f_{j}\right)(x)\right|^{2}\right)^{1 / 2}>\lambda\right\}\right) \\
& \leq w\left(\left\{x \in B:\left(\sum_{j}\left|\mathcal{S}_{\alpha}\left(f_{j}^{0}\right)(x)\right|^{2}\right)^{1 / 2}>\frac{\lambda}{2}\right\}\right) \\
& \quad+w\left(\left\{x \in B:\left(\sum_{j}\left|\mathcal{S}_{\alpha}\left(f_{j}^{\infty}\right)(x)\right|^{2}\right)^{1 / 2}>\frac{\lambda}{2}\right\}\right) \\
& =I_{1}^{\prime}+I_{2}^{\prime} .
\end{aligned}
$$

Theorem B and inequality (20) imply

$$
\begin{aligned}
I_{1}^{\prime} & \leq \frac{2}{\lambda} \cdot\left\|\left(\sum_{j}\left|s_{\alpha}\left(f_{j}^{0}\right)\right|^{2}\right)^{1 / 2}\right\|_{W L_{w}^{1}} \\
& \leq \frac{C}{\lambda} \cdot\left(\int_{2 B}\left(\sum_{j}\left|f_{j}(x)\right|^{2}\right)^{1 / 2} w(x) d x\right) \\
& \leq \frac{C \cdot w(2 B)^{\kappa}}{\lambda}\left\|\left(\sum_{j}\left|f_{j}\right|^{2}\right)^{1 / 2}\right\| \\
& \leq \frac{C \cdot w(B)^{\kappa}}{\lambda}\left\|\left(\sum_{j}\left|f_{j}\right|^{2}\right)^{1 / 2}\right\|_{L^{1, \kappa}(w)} .
\end{aligned}
$$

We now turn to deal with the other term $I_{2}^{\prime}$. In the proof of Theorem 1, we have already shown that, for any $x \in B$ (see (32)),

$$
\begin{aligned}
& \left(\sum_{j}\left|\mathcal{S}_{\alpha}\left(f_{j}^{\infty}\right)(x)\right|^{2}\right)^{1 / 2} \\
& \quad \leq C \sum_{\ell=1}^{\infty} \frac{1}{\left|2^{\ell+1} B\right|} \int_{2^{\ell+1} B}\left(\sum_{j}\left|f_{j}(z)\right|^{2}\right)^{1 / 2} d z .
\end{aligned}
$$

It follows directly from the $A_{1}$ condition that

$$
\begin{aligned}
& \left(\sum_{j}\left|\mathcal{S}_{\alpha}\left(f_{j}^{\infty}\right)(x)\right|^{2}\right)^{1 / 2} \\
& \quad \leq C \sum_{\ell=1}^{\infty} \frac{\operatorname{ess~inf}_{z \in 2^{\ell+1} B} w(z)}{w\left(2^{\ell+1} B\right)} \int_{2^{\ell+1} B}\left(\sum_{j}\left|f_{j}(z)\right|^{2}\right)^{1 / 2} d z
\end{aligned}
$$

$$
\begin{aligned}
& \leq C \sum_{\ell=1}^{\infty} \frac{1}{w\left(2^{\ell+1} B\right)} \int_{2^{\ell+1} B}\left(\sum_{j}\left|f_{j}(z)\right|^{2}\right)^{1 / 2} w(z) d z \\
& \leq C\left\|\left(\sum_{j}\left|f_{j}\right|^{2}\right)^{1 / 2}\right\|_{L^{1, \kappa}(w)} \sum_{\ell=1}^{\infty} \frac{1}{w\left(2^{\ell+1} B\right)^{1-\kappa}} .
\end{aligned}
$$

In addition, since $w \in A_{1} \subset A_{\infty}$, then, by inequality (21), we can see that, for all $x \in B$,

$$
\begin{aligned}
\left(\sum_{j}\left|\mathcal{S}_{\alpha}\left(f_{j}^{\infty}\right)(x)\right|^{2}\right)^{1 / 2} \\
\leq C\left\|\left(\sum_{j}\left|f_{j}\right|^{2}\right)^{1 / 2}\right\|_{L^{1, \kappa}(w)} \\
\cdot \frac{1}{w(B)^{1-\kappa}} \sum_{\ell=1}^{\infty} \frac{w(B)^{1-\kappa}}{w\left(2^{\ell+1} B\right)^{1-\kappa}} \\
\leq C\left\|\left(\sum_{j}\left|f_{j}\right|^{2}\right)^{1 / 2}\right\| \\
\cdot \frac{1}{w(B)^{1-\kappa}} \sum_{\ell=1}^{\infty}\left(\frac{|B|}{\left|2^{\ell+1} B\right|}\right)^{\delta^{*} \cdot(1-\kappa)} \\
\leq C\left\|\left(\sum_{j}\left|f_{j}\right|^{2}\right)^{1 / 2}\right\| \|_{L^{1, \kappa}(w)} \cdot \frac{1}{w(B)^{1-\kappa}},
\end{aligned}
$$

where in the last inequality we have used the fact that $\delta^{*} \cdot(1-$ $\kappa)>0$. If $\left\{x \in B:\left(\sum_{j}\left|\mathcal{S}_{\alpha}\left(f_{j}^{\infty}\right)(x)\right|^{2}\right)^{1 / 2}>\lambda / 2\right\}=\emptyset$, then the inequality

$$
I_{2}^{\prime} \leq \frac{C \cdot w(B)^{\kappa}}{\lambda}\left\|\left(\sum_{j}\left|f_{j}\right|^{2}\right)^{1 / 2}\right\|_{L^{1, \kappa}(w)}
$$

holds trivially. Now if instead we suppose that

$$
\left\{x \in B:\left(\sum_{j}\left|\mathcal{S}_{\alpha}\left(f_{j}^{\infty}\right)(x)\right|^{2}\right)^{1 / 2}>\frac{\lambda}{2}\right\} \neq \emptyset,
$$

then, by the pointwise inequality (39), we have

$$
\lambda \leq C\left\|\left(\sum_{j}\left|f_{j}\right|^{2}\right)^{1 / 2}\right\|_{L^{1, \kappa}(w)} \cdot \frac{1}{w(B)^{1-\kappa}},
$$

which is equivalent to

$$
w(B) \leq \frac{C \cdot w(B)^{\kappa}}{\lambda}\left\|\left(\sum_{j}\left|f_{j}\right|^{2}\right)^{1 / 2}\right\|_{L^{1, \kappa}(w)} .
$$


Therefore

$$
I_{2}^{\prime} \leq w(B) \leq \frac{C \cdot w(B)^{\kappa}}{\lambda}\left\|\left(\sum_{j}\left|f_{j}\right|^{2}\right)^{1 / 2}\right\|_{L^{1, \kappa}(w)} .
$$

Summing up the above estimates for $I_{1}^{\prime}$ and $I_{2}^{\prime}$ and then taking the supremum over all balls $B \subseteq \mathbb{R}^{n}$ and all $\lambda>0$, we finish the proof of Theorem 2 .

\section{Proofs of Theorems 3 and 4}

Proof of Theorem 3. Let $\left(\sum_{j}\left|f_{j}\right|^{2}\right)^{1 / 2} \in L^{p, \Phi}$ with $1<p<\infty$. For any ball $B=B\left(x_{0}, r\right) \subseteq \mathbb{R}^{n}$ with $x_{0} \in \mathbb{R}^{n}$ and $r>0$, we write $f_{j}=f_{j}^{0}+f_{j}^{\infty}$, where $f_{j}^{0}=f_{j} \cdot \chi_{2 B}, j=1,2, \ldots$ Then we have

$$
\begin{aligned}
& \frac{1}{\Phi(r)^{1 / p}}\left(\int_{B\left(x_{0}, r\right)}\left(\sum_{j}\left|\mathcal{S}_{\alpha}\left(f_{j}\right)(x)\right|^{2}\right)^{p / 2} d x\right)^{1 / p} \\
& \leq \frac{1}{\Phi(r)^{1 / p}}\left(\int_{B\left(x_{0}, r\right)}\left(\sum_{j}\left|\mathcal{S}_{\alpha}\left(f_{j}^{0}\right)(x)\right|^{2}\right)^{p / 2} d x\right)^{1 / p} \\
& \quad+\frac{1}{\Phi(r)^{1 / p}}\left(\int_{B\left(x_{0}, r\right)}\left(\sum_{j}\left|\mathcal{S}_{\alpha}\left(f_{j}^{\infty}\right)(x)\right|^{2}\right)^{p / 2} d x\right)^{1 / p} \\
& =J_{1}+J_{2} .
\end{aligned}
$$

Applying Theorem C and the doubling condition (15), we obtain

$$
\begin{aligned}
J_{1} & \leq \frac{1}{\Phi(r)^{1 / p}}\left\|\left(\sum_{j}\left|\mathcal{S}_{\alpha}\left(f_{j}^{0}\right)\right|^{2}\right)^{1 / 2}\right\|_{L^{p}} \\
& \leq C \cdot \frac{1}{\Phi(r)^{1 / p}}\left(\int_{2 B}\left(\sum_{j}\left|f_{j}(x)\right|^{2}\right)^{p / 2} d x\right)^{1 / p} \\
& \leq C\left\|\left(\sum_{j}\left|f_{j}\right|^{2}\right)^{1 / 2}\right\|_{L^{p, \Phi}} \cdot \frac{\Phi(2 r)^{1 / p}}{\Phi(r)^{1 / p}} \\
& \leq C\left\|\left(\sum_{j}\left|f_{j}\right|^{2}\right)^{1 / 2}\right\|_{L^{p, \Phi}} .
\end{aligned}
$$

We now turn to estimate the other term $J_{2}$. We first use inequality (32) and Hölder's inequality to obtain

$$
\begin{aligned}
& \left(\sum_{j}\left|\mathcal{S}_{\alpha}\left(f_{j}^{\infty}\right)(x)\right|^{2}\right)^{1 / 2} \\
& \leq C \sum_{\ell=1}^{\infty} \frac{1}{\left|B\left(x_{0}, 2^{\ell+1} r\right)\right|} \int_{2^{\ell+1} B}\left(\sum_{j}\left|f_{j}(z)\right|^{2}\right)^{1 / 2} d z \\
& \leq C \sum_{\ell=1}^{\infty} \frac{1}{\left|B\left(x_{0}, 2^{\ell+1} r\right)\right|^{1 / p}}\left(\int_{2^{\ell+1} B}\left(\sum_{j}\left|f_{j}(z)\right|^{2}\right)^{p / 2} d z\right)^{1 / p} \\
& \leq C\left\|\left(\sum_{j}\left|f_{j}\right|^{2}\right)^{1 / 2}\right\| \sum_{L^{p, \Phi}}^{\infty} \frac{\Phi\left(2^{\ell+1} r\right)^{1 / p}}{\left|B\left(x_{0}, 2^{\ell+1} r\right)\right|^{1 / p}}
\end{aligned}
$$

Hence

$$
\begin{aligned}
J_{2} \leq & C\left(\sum_{j}\left|f_{j}\right|^{2}\right)^{1 / 2} \|_{L^{p, \Phi}} \\
& \cdot \sum_{\ell=1}^{\infty} \frac{\left|B\left(x_{0}, r\right)\right|^{1 / p}}{\Phi(r)^{1 / p}} \cdot \frac{\Phi\left(2^{\ell+1} r\right)^{1 / p}}{\left|B\left(x_{0}, 2^{\ell+1} r\right)\right|^{1 / p}} .
\end{aligned}
$$

Since $1 \leq D(\Phi)<2^{n}$, then, by using the doubling condition (15) of $\Phi$, we know

$$
\begin{gathered}
\sum_{\ell=1}^{\infty} \frac{\left|B\left(x_{0}, r\right)\right|^{1 / p}}{\Phi(r)^{1 / p}} \cdot \frac{\Phi\left(2^{\ell+1} r\right)^{1 / p}}{\left|B\left(x_{0}, 2^{\ell+1} r\right)\right|^{1 / p}} \\
\leq C \sum_{\ell=1}^{\infty}\left(\frac{D(\Phi)}{2^{n}}\right)^{(\ell+1) / p} \\
\leq C .
\end{gathered}
$$

Therefore

$$
J_{2} \leq C\left\|\left(\sum_{j}\left|f_{j}\right|^{2}\right)^{1 / 2}\right\|_{L^{p, \Phi}}
$$

Combining the above estimates for $J_{1}$ and $J_{2}$ and then taking the supremum over all balls $B=B\left(x_{0}, r\right) \subseteq \mathbb{R}^{n}$, we complete the proof of Theorem 3 .

Proof of Theorem 4. Let $\left(\sum_{j}\left|f_{j}\right|^{2}\right)^{1 / 2} \in L^{1, \Phi}$. For each fixed ball $B=B\left(x_{0}, r\right) \subseteq \mathbb{R}^{n}$, we again decompose $f_{j}$ as $f_{j}=f_{j}^{0}+$ 
$f_{j}^{\infty}$, where $f_{j}^{0}=f_{j} \cdot \chi_{2 B}, j=1,2, \ldots$ For any given $\lambda>0$, then we write

$$
\begin{aligned}
& \left|\left\{x \in B:\left(\sum_{j}\left|\mathcal{S}_{\alpha}\left(f_{j}\right)(x)\right|^{2}\right)^{1 / 2}>\lambda\right\}\right| \\
& \leq\left|\left\{x \in B:\left(\sum_{j}\left|\mathcal{S}_{\alpha}\left(f_{j}^{0}\right)(x)\right|^{2}\right)^{1 / 2}>\frac{\lambda}{2}\right\}\right| \\
& \quad+\left|\left\{x \in B:\left(\sum_{j}\left|\mathcal{S}_{\alpha}\left(f_{j}^{\infty}\right)(x)\right|^{2}\right)^{1 / 2}>\frac{\lambda}{2}\right\}\right| \\
& =J_{1}^{\prime}+J_{2}^{\prime} .
\end{aligned}
$$

Theorem D and the doubling condition (15) imply

$$
\begin{aligned}
J_{1}^{\prime} & \leq \frac{2}{\lambda} \cdot\left\|\left(\sum_{j}\left|\mathcal{S}_{\alpha}\left(f_{j}^{0}\right)\right|^{2}\right)^{1 / 2}\right\|_{W L^{1}} \\
& \leq \frac{C}{\lambda} \cdot\left(\int_{2 B}\left(\sum_{j}\left|f_{j}(x)\right|^{2}\right)^{1 / 2} d x\right) \\
& \leq \frac{C \cdot \Phi(2 r)}{\lambda}\left\|\left(\sum_{j}\left|f_{j}\right|^{2}\right)^{1 / 2}\right\|_{L^{1, \Phi}} \\
& \leq \frac{C \cdot \Phi(r)}{\lambda}\left\|\left(\sum_{j}\left|f_{j}\right|^{2}\right)^{1 / 2}\right\|_{L^{1, \Phi}} .
\end{aligned}
$$

We turn our attention to the estimate of $J_{2}^{\prime}$. Using the preceding estimate (32), we can deduce that, for all $x \in$ $B\left(x_{0}, r\right)$,

$$
\begin{aligned}
& \left(\sum_{j}\left|\delta_{\alpha}\left(f_{j}^{\infty}\right)(x)\right|^{2}\right)^{1 / 2} \\
& \leq C \sum_{\ell=1}^{\infty} \frac{1}{\left|B\left(x_{0}, 2^{\ell+1} r\right)\right|} \int_{2^{\ell+1} B}\left(\sum_{j}\left|f_{j}(z)\right|^{2}\right)^{1 / 2} d z \\
& \leq C\left\|\left(\sum_{j}\left|f_{j}\right|^{2}\right)^{1 / 2}\right\| \sum_{L^{1, \Phi} \ell=1}^{\infty} \frac{\Phi\left(2^{\ell+1} r\right)}{\left|B\left(x_{0}, 2^{\ell+1} r\right)\right|} \\
& \leq C\left\|\left(\sum_{j}\left|f_{j}\right|^{2}\right)^{1 / 2}\right\|_{L^{1, \Phi}} \cdot \frac{\Phi(r)}{\left|B\left(x_{0}, r\right)\right|} \\
& \quad \times \sum_{\ell=1}^{\infty} \frac{\left|B\left(x_{0}, r\right)\right|}{\Phi(r)} \cdot \frac{\Phi\left(2^{\ell+1} r\right)}{\left|B\left(x_{0}, 2^{\ell+1} r\right)\right|} .
\end{aligned}
$$

Note that $1 \leq D(\Phi)<2^{n}$. Arguing as in the proof of (49), we can get

$$
\begin{gathered}
\sum_{\ell=1}^{\infty} \frac{\left|B\left(x_{0}, r\right)\right|}{\Phi(r)} \cdot \frac{\Phi\left(2^{\ell+1} r\right)}{\left|B\left(x_{0}, 2^{\ell+1} r\right)\right|} \\
\leq \sum_{\ell=1}^{\infty}\left(\frac{D(\Phi)}{2^{n}}\right)^{\ell+1} \\
\leq C .
\end{gathered}
$$

Hence, for any $x \in B\left(x_{0}, r\right)$,

$$
\begin{aligned}
& \left(\sum_{j}\left|\mathcal{S}_{\alpha}\left(f_{j}^{\infty}\right)(x)\right|^{2}\right)^{1 / 2} \\
& \quad \leq C\left\|\left(\sum_{j}\left|f_{j}\right|^{2}\right)^{1 / 2}\right\|_{L^{1, \Phi}} \cdot \frac{\Phi(r)}{\left|B\left(x_{0}, r\right)\right|} .
\end{aligned}
$$

If $\left\{x \in B:\left(\sum_{j}\left|\mathcal{S}_{\alpha}\left(f_{j}^{\infty}\right)(x)\right|^{2}\right)^{1 / 2}>\lambda / 2\right\}=\emptyset$, then the inequality

$$
J_{2}^{\prime} \leq \frac{C \cdot \Phi(r)}{\lambda}\left\|\left(\sum_{j}\left|f_{j}\right|^{2}\right)^{1 / 2}\right\|_{L^{1, \Phi}}
$$

holds trivially. Now we may suppose that

$$
\left\{x \in B:\left(\sum_{j}\left|\delta_{\alpha}\left(f_{j}^{\infty}\right)(x)\right|^{2}\right)^{1 / 2}>\frac{\lambda}{2}\right\} \neq \emptyset .
$$

Then, by the pointwise inequality (55), we can see

$$
\lambda \leq C\left\|\left(\sum_{j}\left|f_{j}\right|^{2}\right)^{1 / 2}\right\|_{L^{1, \Phi}} \cdot \frac{\Phi(r)}{\left|B\left(x_{0}, r\right)\right|},
$$

which is equivalent to

$$
\left|B\left(x_{0}, r\right)\right| \leq \frac{C \cdot \Phi(r)}{\lambda}\left\|\left(\sum_{j}\left|f_{j}\right|^{2}\right)^{1 / 2}\right\|_{L^{1, \Phi}} .
$$

Therefore

$$
J_{2}^{\prime} \leq\left|B\left(x_{0}, r\right)\right| \leq \frac{C \cdot \Phi(r)}{\lambda}\left\|\left(\sum_{j}\left|f_{j}\right|^{2}\right)^{1 / 2}\right\|_{L^{1, \Phi}} .
$$

Summing up the above estimates for $J_{1}^{\prime}$ and $J_{2}^{\prime}$ and then taking the supremum over all balls $B=B\left(x_{0}, r\right) \subseteq \mathbb{R}^{n}$ and all $\lambda>0$, we conclude the proof of Theorem 4 .

\section{Conflict of Interests}

The author declares that there is no conflict of interests regarding the publication of this paper. 


\section{References}

[1] M. Wilson, “The intrinsic square function," Revista Matematica Iberoamericana, vol. 23, no. 3, pp. 771-791, 2007.

[2] M. Wilson, Weighted Littlewood-Paley Theory and ExponentialSquare Integrability, vol. 1924 of Lecture Notes in Math, Springer, 2007.

[3] C. B. Morrey, "On the solutions of quasi-linear elliptic partial differential equations," Transactions of the American Mathematical Society, vol. 43, pp. 126-166, 1938.

[4] D. R. Adams, "A note on Riesz potentials," Duke Mathematical Journal, vol. 42, no. 4, pp. 765-778, 1975.

[5] F. Chiarenza and M. Frasca, "Morrey spaces and HardyLittlewood maximal function," Rendiconti di Matematica e delle sue Applicazioni, vol. 7, no. 3-4, pp. 273-279, 1987.

[6] J. Peetre, "On the theory of $L_{p, \lambda}$ spaces," Journal of Functional Analysis, vol. 4, no. 1, pp. 71-87, 1969.

[7] T. Mizuhara, "Boundedness of some classical operators on generalized Morrey spaces, harmonic analysis," in ICM-90 Satellite Conference Proceedings, pp. 183-189, Springer, Tokyo, Japan, 1991.

[8] V. S. Guliyev, "Boundedness of the maximal, potential and singular operators in the generalized Morrey spaces," Journal of Inequalities and Applications, vol. 2009, Article ID 503948, 2009.

[9] V. S. Guliyev, S. S. Aliyev, and T. Karaman, "Boundedness of a class of sublinear operators and their commutators on generalized Morrey spaces," Abstract and Applied Analysis, vol. 2011, Article ID 356041, 18 pages, 2011.

[10] V. S. Guliyev, S. S. Aliyev, T. Karaman, and P. S. Shukurov, "Boundedness of sublinear operators and commutators on generalized Morrey spaces," Integral Equations and Operator Theory, vol. 71, no. 3, pp. 327-355, 2011.

[11] S. Z. Lu, D. C. Yang, and Z. S. Zhou, "Sublinear operators with rough kernel on generalized Morrey spaces," Hokkaido Mathematical Journal, vol. 27, no. 1, pp. 219-232, 1998.

[12] E. Nakai, "Hardy-Littlewood maximal operator, singular integral operators and Riesz potentials on generalized Morrey spaces," Mathematische Nachrichten, vol. 166, no. 1, pp. 95-103, 1994.

[13] Y. Komori and S. Shirai, "Weighted Morrey spaces and a singular integral operator," Mathematische Nachrichten, vol. 282, no. 2, pp. 219-231, 2009.

[14] H. Wang, "Intrinsic square functions on the weighted Morrey spaces," Journal of Mathematical Analysis and Applications, vol. 396, no. 1, pp. 302-314, 2012.

[15] H. Wang, "Weak type estimates for intrinsic square functions on weighted Morrey spaces," Analysis in Theory and Applications, vol. 29, pp. 104-119, 2013.

[16] H. Wang, "Boundedness of intrinsic square functions on generalized Morrey spaces," The Georgian Mathematical Journal, vol. 21, 2014. 


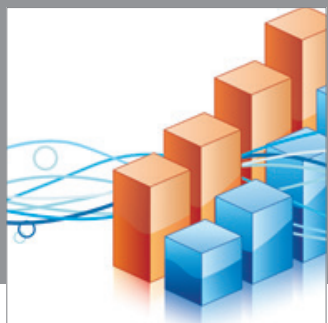

Advances in

Operations Research

mansans

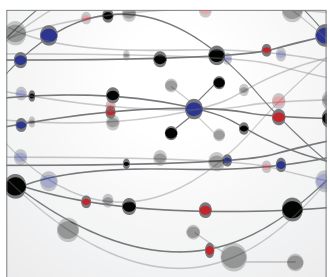

The Scientific World Journal
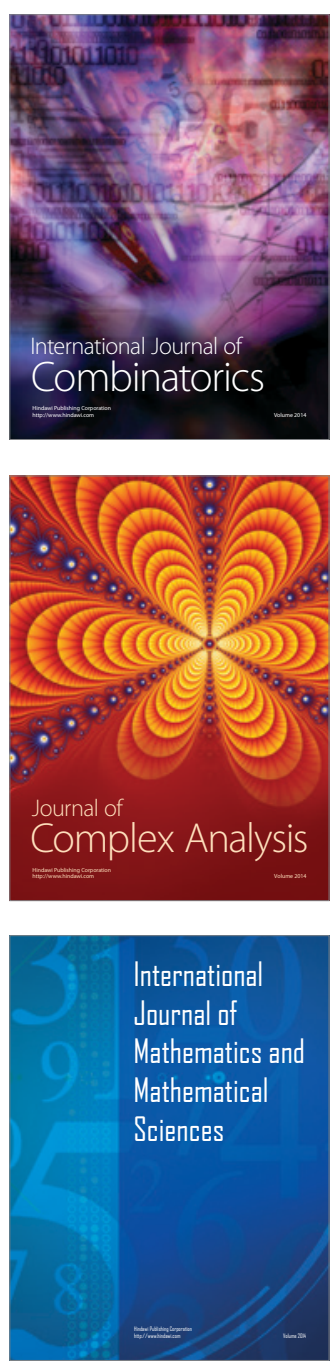
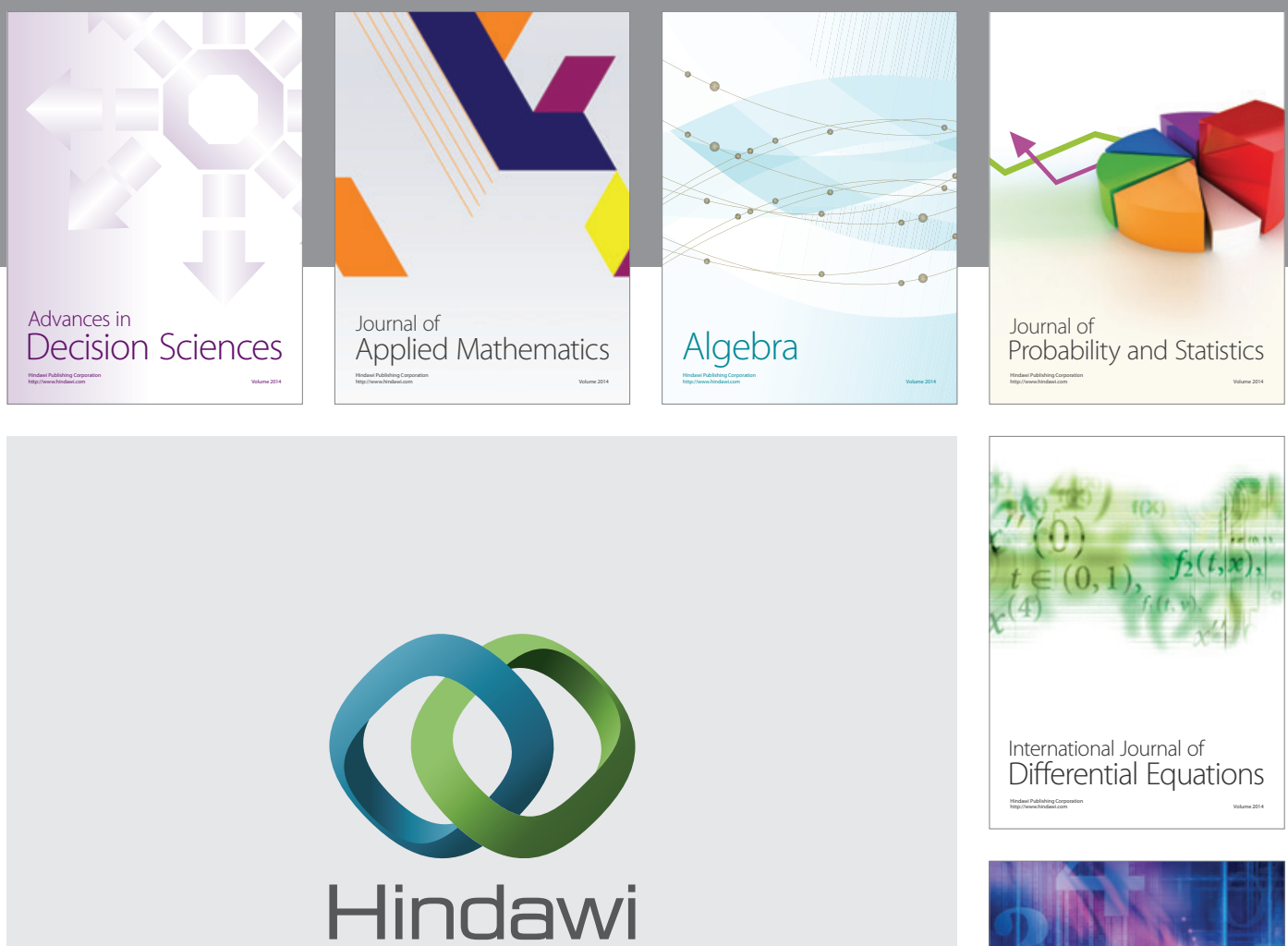

Submit your manuscripts at http://www.hindawi.com
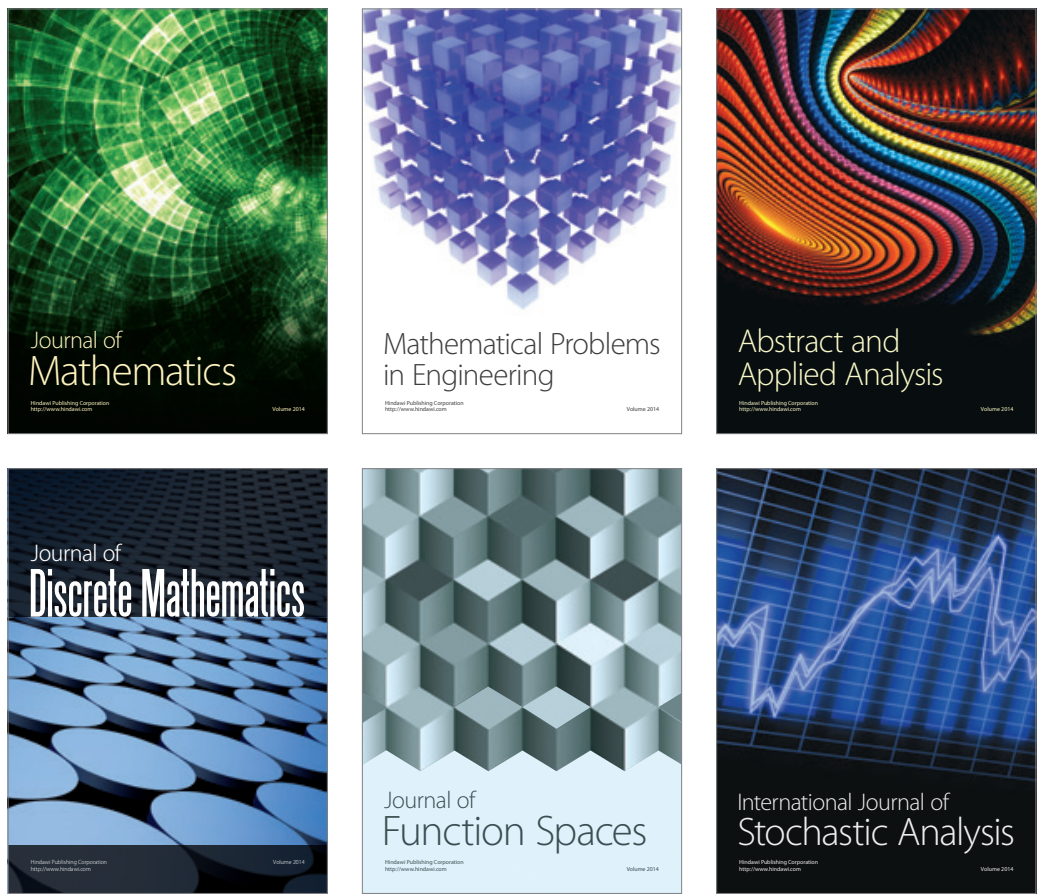

Journal of

Function Spaces

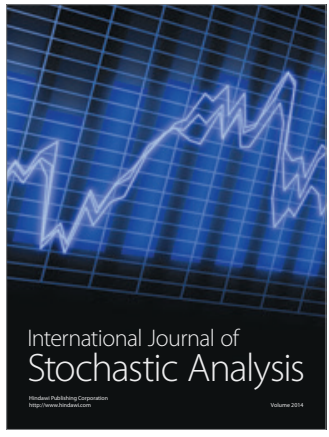

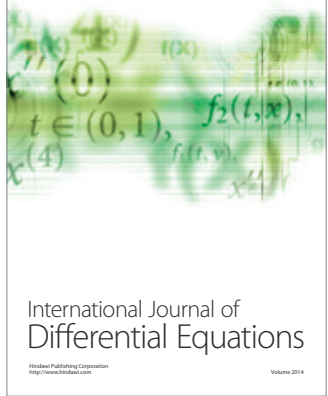
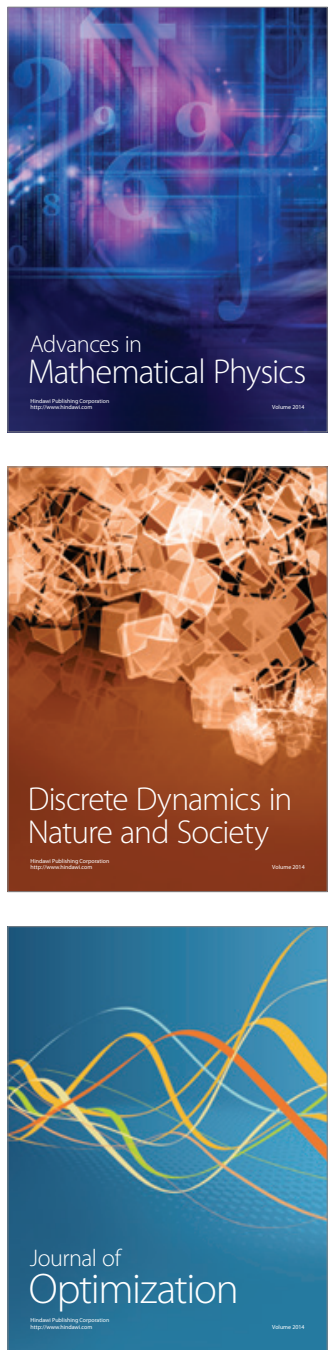